\title{
Two-Dimensional Rotorcraft Downwash Flow Field Measurements by Lidar-Based Wind Scanners with Agile Beam Steering
}

Sjöholm, Mikael; Angelou, Nikolas; Hansen, Per; Hansen, Kasper Hjorth; Mikkelsen, Torben; Haga, Steinar; Silgjerd, Jon Arne; Starsmore, Neil

Published in:

Journal of Atmospheric and Oceanic Technology

Link to article, DOI:

10.1175/JTECH-D-13-00010.1

Publication date:

2014

Document Version

Publisher's PDF, also known as Version of record

Link back to DTU Orbit

Citation (APA):

Sjöholm, M., Angelou, N., Hansen, P., Hansen, K. H., Mikkelsen, T., Haga, S., Silgjerd, J. A., \& Starsmore, N. (2014). Two-Dimensional Rotorcraft Downwash Flow Field Measurements by Lidar-Based Wind Scanners with Agile Beam Steering. Journal of Atmospheric and Oceanic Technology, 31(4), 930-937. https://doi.org/10.1175/JTECH-D-13-00010.1

\section{General rights}

Copyright and moral rights for the publications made accessible in the public portal are retained by the authors and/or other copyright owners and it is a condition of accessing publications that users recognise and abide by the legal requirements associated with these rights.

- Users may download and print one copy of any publication from the public portal for the purpose of private study or research.

- You may not further distribute the material or use it for any profit-making activity or commercial gain

- You may freely distribute the URL identifying the publication in the public portal 


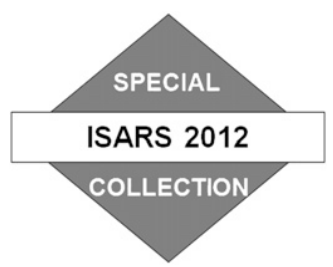

\title{
${ }^{\curvearrowright}$ Two-Dimensional Rotorcraft Downwash Flow Field Measurements by Lidar-Based Wind Scanners with Agile Beam Steering
}

\author{
Mikael Sjöholm, Nikolas Angelou, Per Hansen, Kasper Huorth Hansen, \\ AND TORBEN MIKKELSEN \\ Department of Wind Energy, Technical University of Denmark, Roskilde, Denmark \\ Steinar Haga AND Jon ARne SilgJerd \\ Automasjon og Data AS, Sandnes, Norway \\ NEIL STARSMORE \\ Starsmore Ltd., Blackwater Hollow, Rookley, United Kingdom
}

(Manuscript received 8 January 2013, in final form 12 October 2013)

\begin{abstract}
A major risk to helicopters is the unexpected encounter of degraded visual environments in close-to-ground operations, where a loss of visibility often is caused by clouds of dust (brownout) or snow (whiteout) stirred up by intense downwash. The understanding of the phenomenon is limited, and there is a need for instruments that can measure flow fields on scales larger than a few meters with good resolution. This paper reports on the use of synchronized continuous-wave Doppler lidars for rotorcraft downwash flow field studies.

Built from a modified ZephIR wind lidar and a double-prism arrangement for agile beam steering, a wind scanner-WindScanner-has been developed at the Department of Wind Energy at the Technical University of Denmark (DTU) Ris $\varnothing$ campus. The WindScanner measures the line-of-sight component of the airflow remotely and by rapid steering, the line-of-sight direction and the focus position; all points in space within a cone with a full opening angle of $120^{\circ}$ can be reached from about $8 \mathrm{~m}$ out to some hundred meters depending on the range resolution required.

The first two-dimensional mean wind fields measured in a horizontal plane and in a vertical plane below a hovering search and rescue helicopter are presented. Since the line-of-sight directions of the two synchronized WindScanners were scanned within the plane of interest, the influence of the wind component perpendicular to the plane was avoided. The results also demonstrate the possibilities within less demanding flows encountered within complex terrain and wind-energy-related research for which the WindScanner technology primarily has been developed.
\end{abstract}

\section{Introduction}

A major hazardous risk to helicopters is the unexpected encounter of degraded visual environments (DVEs) in close-to-ground operations, where a loss of visibility often

\footnotetext{
¿ Denotes Open Access content.
}

Corresponding author address: Mikael Sjöholm, Section of Test and Measurements, Department of Wind Energy, Technical University of Denmark, Risø Campus, Frederiksborgvej 399, DK-4000 Roskilde, Denmark.

E-mail: misj@dtu.dk is dangerously detrimental. The rotor downwash flow can cause such conditions as low visibility by stirring up sand and dust into an obscuring cloud surrounding the rotorcraft. This particular phenomenon is termed "brownout" and its counterparts occurring above water and above snow are termed "whiteout."

Despite ongoing developments of various support aids for pilots in DVEs, including visual displays but also innovative tactile display techniques (Jansen et al. 2008) of information gathered from traditional flight parameters or enhanced artificial images in, for instance, the terahertz range (Fiorino et al. 2012) or from range-gated detection of active pulsed illumination (Roy et al. 2012), 
there is still a lack of fundamental knowledge about the brownout process itself.

In particular, knowledge about the influence of various rotorcraft design parameters on the severity of brownouts is limited, as pointed out by Milluzzo and Leishman (2010), who by analyzing photographs and videographs of brownout events found some clues to which parameters are of importance for the severity of the events. Computational fluid dynamics (CFD) calculations have also previously been used in order to gain insight into the brownout phenomenon, but there is only limited experimental data from full-scale flight tests for code verification, as mentioned by Wadcock et al. (2008), who imaged the movements of tufts on a grid below a hovering helicopter as an experimental indicator of the downwash footprint.

However, for some time there has been a vision about rapid three-dimensional measurements of turbulent flows in complex environments (Mikkelsen et al. 2008). Now a ground-based remote-sensing wind-scanning instrument-WindScanner-has been developed at the Department of Wind Energy at the Technical University of Denmark (DTU) Ris $\varnothing$ campus in Denmark. The technology has primarily been developed for studies of the three-dimensional flow fields around wind turbines and the wind fields around complex natural and man-made structures. Here, however, the potential of the technology for full-scale rotorcraft flow field studies is outlined and a proof-of-concept trial in the extremely turbulent and complex downwash flow below a search and rescue (SAR) helicopter is presented.

\section{The measurement technology}

The WindScanner research infrastructure consists of both continuous-wave short-range and pulsed longrange remote sensing wind lidar systems. The shortrange WindScanner technology is based on a coherent continuous-wave wind lidar instrument-that is, a ZephIR 150 manufactured by Natural Power (Malvern, United Kingdom), which is designed based on the principles outlined in Karlsson et al. (2000), although it has been modified according to the description below. For agile beam scanning, a patented double-prism arrangement was developed in cooperation with the industrial design firm IPU (Kongens Lyngby, Denmark). This arrangement allows for rapid and synchronized steering of the pointing directions of a focused laser beam by rotating two wedge-shaped optical prisms and steering of the focus position by a motor moving an optical fiber relative to the focusing lens in each WindScanner. Thus, all locations within a cone with a full opening angle of $120^{\circ}$ can be reached from about
$8 \mathrm{~m}$ out to some hundred meters depending on the range resolution required.

The wind lidar measures a line-averaged (Sjöholm et al. 2009) line-of-sight component of the wind by coherently detecting the beat frequency between the transmitted light and the Doppler-shifted light backscattered from naturally occurring aerosols in the moving air. Typically, the encountered Doppler shifts are in the megahertz frequency range, since a line-of-sight wind speed of $1 \mathrm{~m} \mathrm{~s}^{-1}$ corresponds to $1.28 \mathrm{MHz}$ at the particular wavelength of $1565 \mathrm{~nm}$ used. The detected signal is sampled at $100 \mathrm{MHz}$ and from 512 samples a fast Fourier transform (FFT) calculates spectra extending up to $50 \mathrm{MHz}$, which allows for wind speed assessment up to about $39.1 \mathrm{~m} \mathrm{~s}^{-1}$. However, in order to distinguish between winds blowing away from or toward the instrument, an acousto-optic modulator shifting the transmitted light by $27 \mathrm{MHz}$ is incorporated, which limits the detectable line-of-sight wind speed on the positive side to $18 \mathrm{~m} \mathrm{~s}^{-1}$. In this study, typically 500 such spectra were averaged to reduce the noise contribution, rendering a stream of averaged spectra at a rate of $390.625 \mathrm{~Hz}$.

The line-averaging sampling profile of such a focused coherent lidar has been characterized in detail (Angelou et al. 2012b), and the velocity estimation for conically scanning ZephIRs has previously been verified (Gottschall et al. 2012). However, the particular instruments used have a larger effective beam radius (28 instead of $20 \mathrm{~mm}$ ) on the telescope, which means that a better confinement of the sampling volume along the beam is achieved. For the instrument used, the full width at half maximum (FWHM) of the Lorentz-shaped lineof-sight sampling profile is $12.6 \mathrm{~m}$ at a measurement distance of $100 \mathrm{~m}$ and since the width depends quadratically on the distance between the instrument and the center of the measurement volume, the corresponding width has decreased to $3.2 \mathrm{~m}$ at a distance of $50 \mathrm{~m}$, and at a distance of $10 \mathrm{~m}$ it is only about $0.1 \mathrm{~m}$.

\section{The experimental setup}

In the beginning of December 2011, a helicopter downwash proof-of-concept field test was performed with two synchronized ground-based short-range WindScanners. They measured the flow field below a hovering SAR helicopter in a horizontal plane close to the ground and in a vertical plane below the helicopter. The particular short-range WindScanners used for measuring the twodimensional domains of two of the wind vector components were the units R2D1 and R2D2. By using two WindScanners and keeping the line-of-sight directions of them inside the planes scanned, the wind components perpendicular to the planes of interest were not influencing the line-of-sight wind speeds measured. 


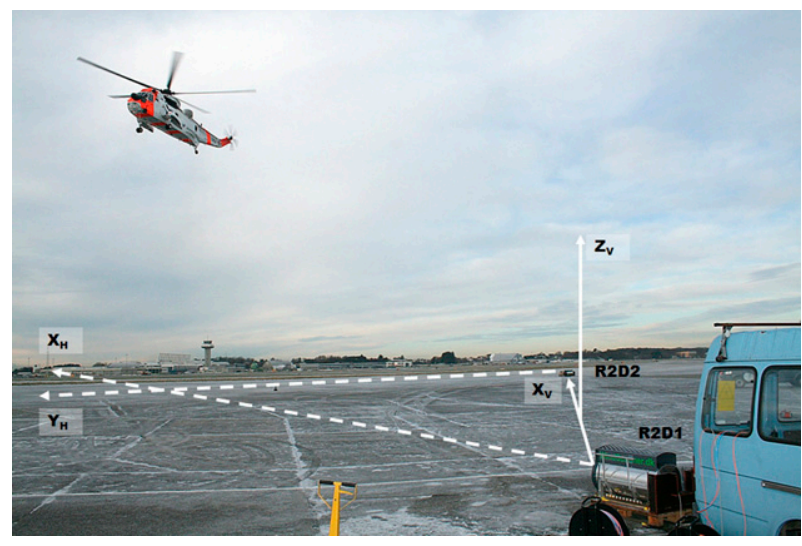

FIG. 1. The image shows the experimental setup for scanning a horizontal plane beneath the hovering helicopter. Note that for scanning the vertical plane, the helicopter was instead hovering above the center of the line between the R2D1 and R2D2 WindScanners. The coordinate system used for the horizontal scan is outlined by dashed lines and index $\mathrm{H}$, whereas the coordinate system used for the vertical scan is outlined by solid lines and index $\mathrm{V}$.

The setups used during the scanning in the horizontal plane and in the vertical plane are outlined in Fig. 1. A special mount with easily adjustable legs was used in order to facilitate measurements close to the ground. With this arrangement, the scanning beam emanated from a height of only $0.32 \mathrm{~m}$ above the EUR-pallet on which the WindScanner was standing. Thus, the scanned horizontal plane $(z=0 \mathrm{~m})$ was about $0.46 \mathrm{~m}$ above the ground. In addition, the EUR-pallet arrangement made the WindScanners easily movable by a lifting fork.

For the scanning of the horizontal plane, the center of the measurement volumes of the two laser beams synchronously traced the trajectory of an Archimedean spiral that spiraled outward from the origin of the coordinate system, through 15 revolutions during $10 \mathrm{~s}$, out to a radius of about $30 \mathrm{~m}$ and then back to the origin during $2 \mathrm{~s}$ along a radial ray crossing the center of the line between the two WindScanners. Along the return line in the scanning pattern, an ultrasonic anemometer sampling at $100 \mathrm{~Hz}$ was manually traversed in order to study the high-frequency content in various local measurement positions.

For the scanning of the vertical plane, the locations of the two WindScanners were the same as for the scanning of the horizontal plane. However, they were directed toward each other with the helicopter hovering above the center of the 78.8-m-long line between them. The center of the measurement volumes of the two laser beams synchronously traced the trajectory of a sinusoidal wave oscillating between ground and a height of $20 \mathrm{~m}$ with 40 periods within a 50-m-long horizontal range centered below the helicopter in $20 \mathrm{~s}$ and then back along a horizontal line to the starting point in $2 \mathrm{~s}$.

\section{Analysis procedure}

The data analysis starts by the synchronization of wind Doppler spectra streamed from the lidars with the corresponding measurement positions in the air calculated from the motor positions sampled by the motion control system. Subsequently, the Doppler spectra are normalized by a noise background spectrum in order to flatten out the spectra, such that structures in the spectra that are due to the measurement system itself become flat in the whitened spectra, as discussed in Angelou et al. (2012a). To increase the apparent spectral signal-to-noise ratio, several spectra are averaged. In this study, the spectra are sorted into grid cells with the size of $2 \mathrm{~m} \times 2 \mathrm{~m}$ in the horizontal scan and into grid cells with the horizontal size of $2 \mathrm{~m}$ and the vertical size of $0.5 \mathrm{~m}$ in the vertical scan, and then the median value in each frequency bin is calculated from all the spectra obtained in each grid cell during each scan, resulting in a socalled median spectrum, with less noise.

The spectrum of the Doppler-shifted backscattered radiation basically describes the velocity distribution within the sampled volume filtered by the Lorentz averaging function (Sjöholm et al. 2009). In the case of a spectrum resulting from a constant steady wind speed with low turbulence and a good signal-to-noise ratio rendering a distinct single narrow peak, the frequency location of that peak is straightforward to find. However, in a turbulent flow below a rotorcraft the peak broadens, as seen in Fig. 2, and it becomes more challenging to estimate the mean wind speed, as discussed in Angelou et al. (2012a). Therefore, a frequency estimation procedure based on a Gaussian-fit algorithm is used in this study as illustrated in Fig. 2, where also the influence of the varying sampling volume can be seen. The left spectrum in Fig. 2 is sampled at a line-of-sight distance of $23.4 \mathrm{~m}$ with a sampling volume of $0.7 \mathrm{~m}$, whereas the right spectrum is sampled at a distance of $55.4 \mathrm{~m}$ with a sampling volume of $3.9 \mathrm{~m}$, which results in a broader spectral distribution due to the rapid spatial variability of the downwash flow.

As long as the two line-of-sight directions are scanned within a common plane-for example, the $x-y$ planeand not coinciding, the combined corresponding twodimensional wind vector components in that plane-that is, $v_{x}$ and $v_{y}$-can be retrieved from the two simultaneously measured line-of-sight wind speeds $v_{1}$ and $v_{2}$, which also has been discussed in, for instance, Newsom et al. (2008). The retrieval was done by Eq. (1), where $k=\cos \left(\alpha_{1}\right) \sin \left(\alpha_{2}\right)-$ $\cos \left(\alpha_{2}\right) \sin \left(\alpha_{1}\right)$ and $\alpha_{1}$ and $\alpha_{2}$ are the polar angles (positive counterclockwise seen from above) from the $x$ axis toward 

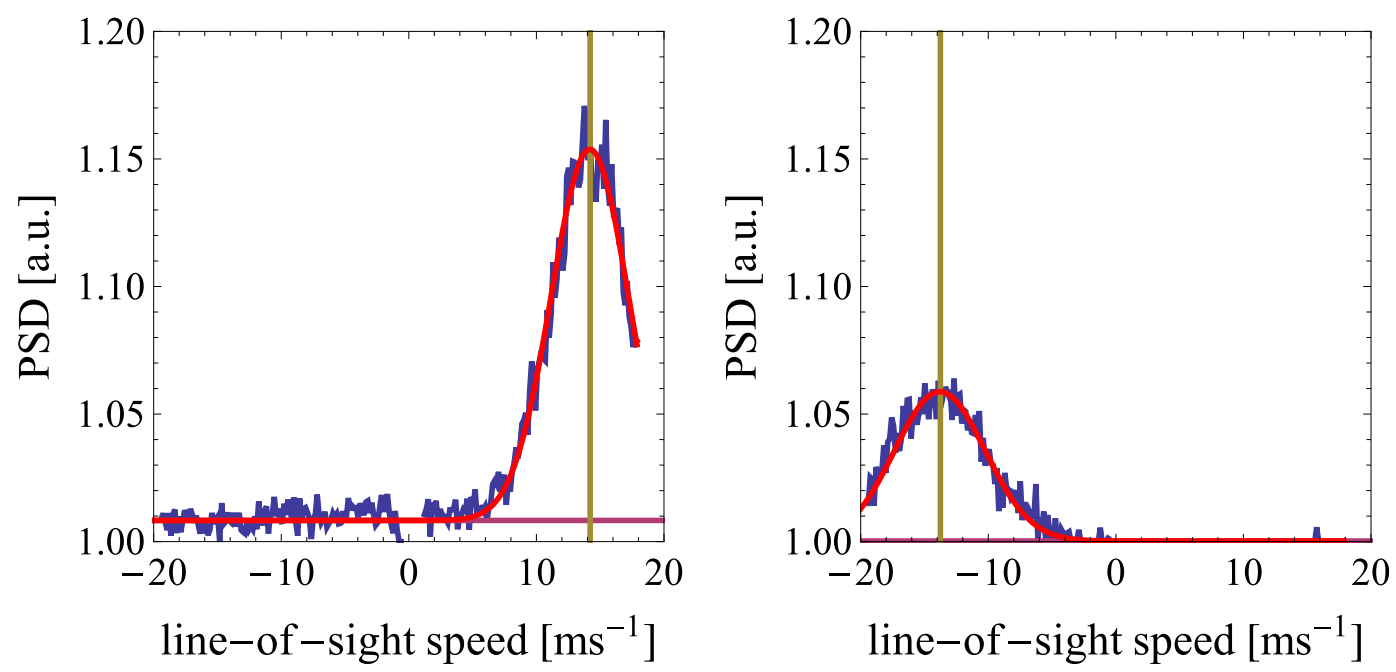

FIG. 2. The spectra from the vertical scan at the location $x=-16 \mathrm{~m}$ close to ground measured (left) by R2D1 at a distance of $23.4 \mathrm{~m}$ with a sampling volume of $0.7 \mathrm{~m}$ and (right) by R2D2 at a distance of $55.4 \mathrm{~m}$ with a sampling volume of $3.9 \mathrm{~m}$. A Gaussian curve was fitted to the spectra as shown and the location of its peak was used as an estimate of the mean speed, which in this particular example was estimated to be 14.2 and $-13.7 \mathrm{~m} \mathrm{~s}^{-1}$, respectively.

the line-of-sight directions going from the center of the measurement volumes toward the instruments,

$$
\left[\begin{array}{l}
v_{x} \\
v_{y}
\end{array}\right]=\frac{1}{k}\left[\begin{array}{cc}
\sin \left(\alpha_{2}\right) & -\sin \left(\alpha_{1}\right) \\
-\cos \left(\alpha_{2}\right) & \cos \left(\alpha_{1}\right)
\end{array}\right]\left[\begin{array}{l}
v_{1} \\
v_{2}
\end{array}\right]
$$

\section{The flow field in a vertical plane below a helicopter}

While the SAR helicopter was hovering at a height of $70 \mathrm{ft}(\sim 21 \mathrm{~m})$ with the front almost aligned with the negative $x$ axis, the downwash was measured in a vertical plane below the helicopter by two WindScanners and the averaged results for a period of $9 \mathrm{~min}$ are presented in Fig. 3. The center of the scanner head of the R2D1 WindScanner was placed at $x=-39.4 \mathrm{~m}$ and $z=0 \mathrm{~m}$, which explains why the sampled component of the wind vector in the bottom-left corner of the top panel in Fig. 3 is positive, since the flow away from the helicopter hovering above approximately $x=0 \mathrm{~m}$ is directed toward the R2D1 WindScanner in that region. Correspondingly, in the bottom-right part, the flow is away from the R2D1 WindScanner, resulting in a negative wind speed component measured by the R2D1 WindScanner.

The corresponding average of the line-of-sight wind component measured by the R2D2 WindScanner placed at $x=39.4 \mathrm{~m}$ and $z=0 \mathrm{~m}$ is displayed in the middle panel in Fig. 3, and the resulting combined vertical twodimensional flow field is presented in the bottom panel in Fig. 3. It can clearly be seen that the mean flow below the rotor is directed downward and confined within a diameter of about $20 \mathrm{~m}$ and that the flow deflects outward as it approaches the ground.

\section{The flow field in a horizontal plane close to ground}

In addition to the measurement of a vertical plane, the helicopter downwash was also measured in a horizontal plane about $0.46 \mathrm{~m}$ above the ground with the helicopter hovering at $90 \mathrm{ft}(\sim 27 \mathrm{~m})$ approximately above the origin of the coordinate system and with the front almost aligned with the $135^{\circ}$ radial ray relative to the $x$ axis. Using Eq. (1) the 10-min-averaged line-of-sight wind components measured by the R2D1 WindScanner placed at $x=-60 \mathrm{~m}$ and $y=0 \mathrm{~m}$ and the R2D2 placed at $x=0 \mathrm{~m}$ and $y=-50 \mathrm{~m}$ were combined into an average two-dimensional flow field in the horizontal plane, as presented in Fig. 4.

It can clearly be seen in Fig. 4 that the flow emanates from a location slightly above and to the right of the center of the plot, where the helicopter was located within about a meter according to the pilot. The $x$ axis in Fig. 4 is approximately pointing toward west-northwest in the direction of $285^{\circ}$ from the north and the average ambient wind was reported to be $2 \mathrm{kt}\left(1 \mathrm{kt}=0.51 \mathrm{~m} \mathrm{~s}^{-1}\right)$ easterly, which contributes to the slight displacement of the downwash. The measured flow field exhibits, as expected, an almost annular shape with some complex structures and maximum speeds at about some $15 \mathrm{~m}$ from the center of the calm area below the helicopter.

As an illustration of the turbulent fluctuations on the time scale of completing a scan pattern-that is, $12 \mathrm{~s}-$ the time evolution of the $x$-axis-parallel component of 

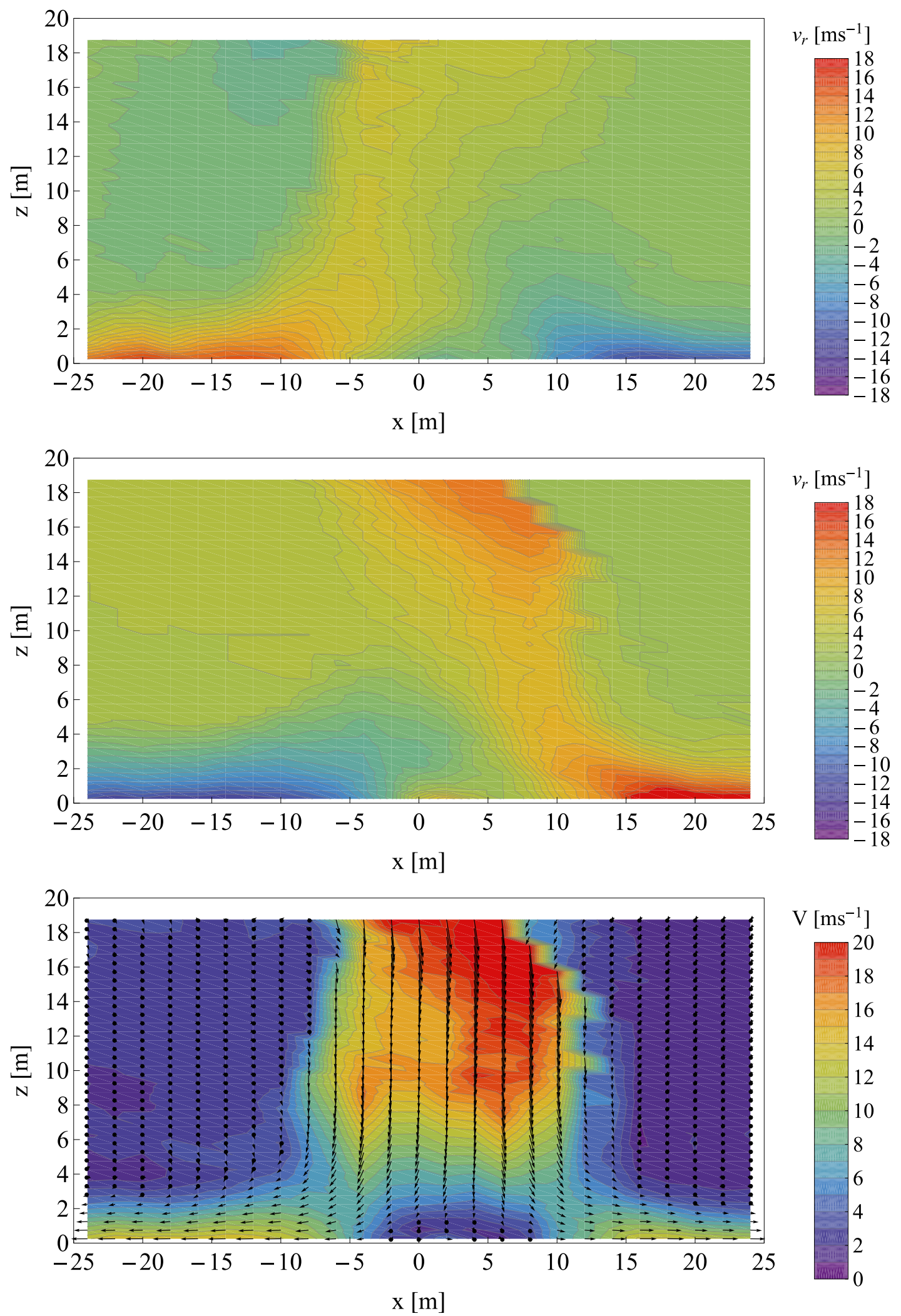

$\mathrm{V}\left[\mathrm{ms}^{-1}\right]$

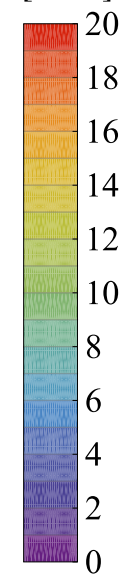

FIG. 3. The 9-min-average line-of-sight wind component $v_{r}$ measured by WindScanner (top) R2D1 and (middle) R2D2 (contours and shading). (bottom) The retrieved two-dimensional $(x-z)$ wind field (vectors, contours, and shading) in a vertical plane beneath the helicopter. 


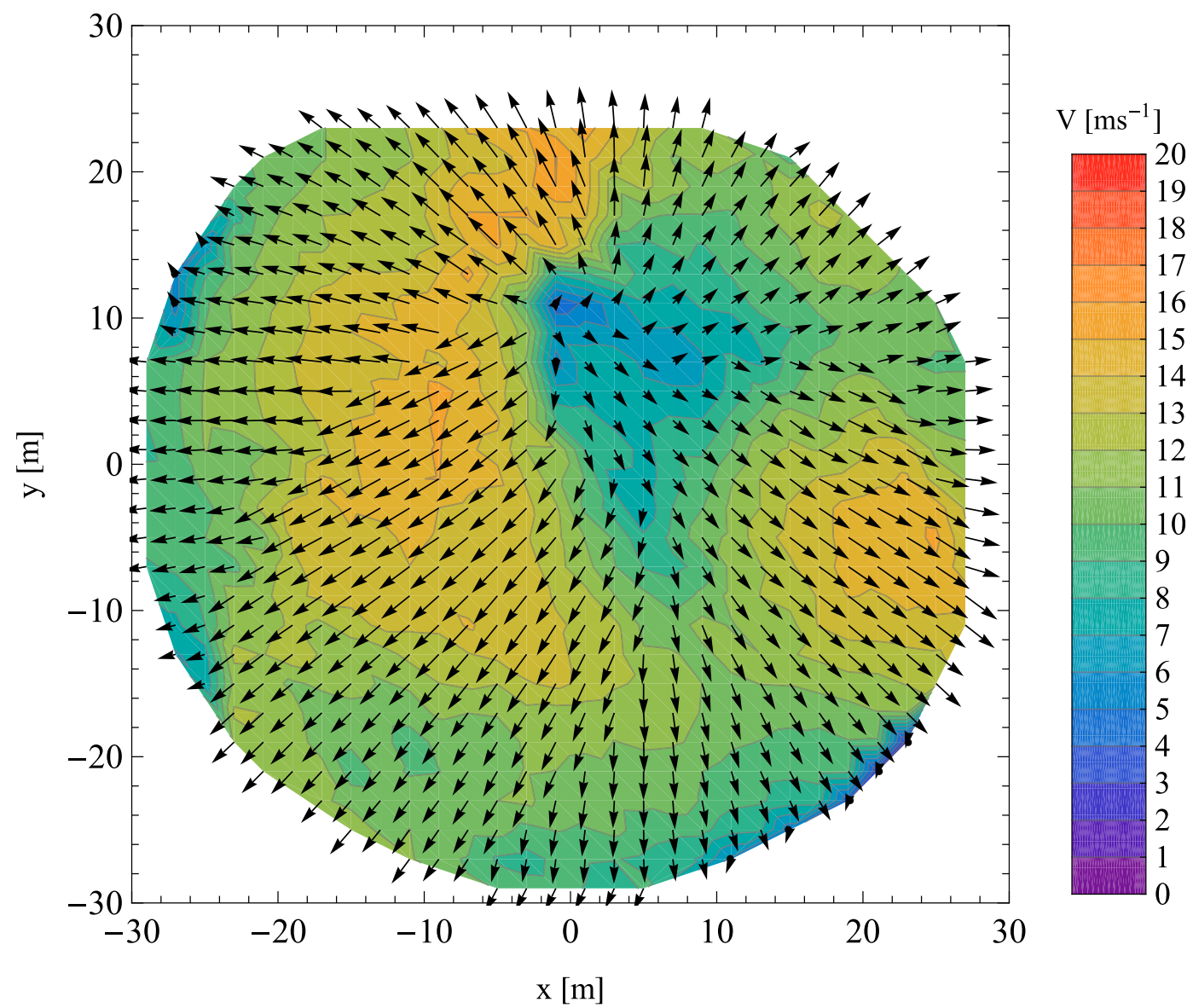

FIG. 4. The 10-min-average two-dimensional $(x-y)$ wind field (vectors, contours, and shading), in a horizontal plane close to ground achieved by combining the line-of-sight wind velocities measured by the two WindScanners.

the two-dimensional velocity field in Fig. 4 along the $x$ axis at $y=0$ is given in Fig. 5 for the time period of 10 min during which the averaging of the mean flow presented in Fig. 4 took place. In Fig. 5, it can be seen that even the 12-s individual scans reveal the main average structure of the flow. However, in order to quantify the influence of the averaging time, a few $x$-axis locations $(20,10,0,-10$, and $-20 \mathrm{~m})$ in Fig. 5 were selected, and the influence of increasing averaging time is shown in Fig. 6. As can be seen, an averaging time of about 2 min was sufficient for obtaining a stable assessment of the average flow in this case.

Along the return line in the scan pattern from the bottom-left corner to the origin $\left(225^{\circ}\right.$ from the $x$ axis) in

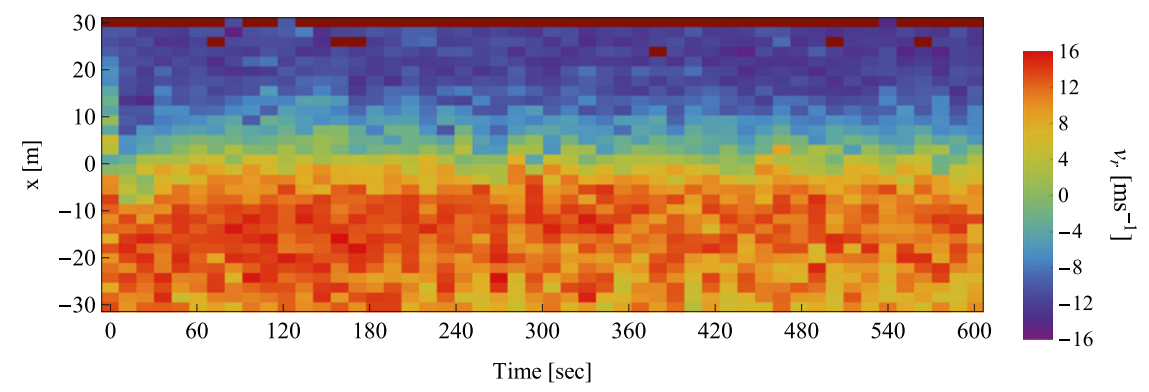

FIG. 5. The time evolution of the $x$-axis-parallel component of the two-dimensional velocity field (shading) in Fig. 4 along the $x$ axis at $y=0$. 


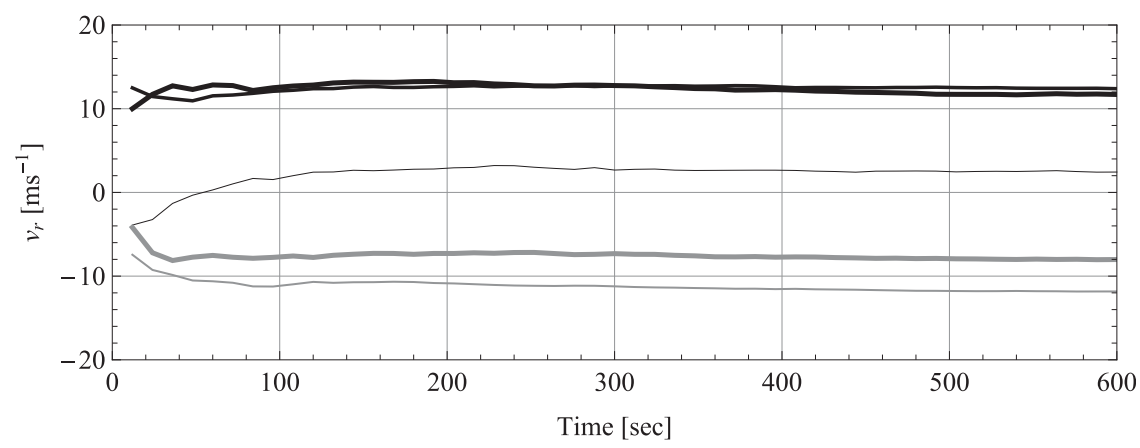

FIG. 6. The wind component in Fig. 5 at $x$ locations $-20,-10,0,10$, and $20 \mathrm{~m}$ (in the order of decreasing line thickness) averaged during increasingly longer averaging periods ranging from 12 to $600 \mathrm{~s}$.

Fig. 4, a comparison with an ultrasonic anemometer could be done. The ultrasonic anemometer stayed in each measurement position for a period of $41 \mathrm{~s}$ (acquiring 4096 samples at $100 \mathrm{~Hz}$ ) and then traversed a distance of $2 \mathrm{~m}$ along the line to the next measurement position. The 15 measurement positions were completed within a period of slightly more than $10 \mathrm{~min}$. During the same period, the WindScanners were scanning the entire horizontal plane and the mean speed in each 1-m-long grid cell was evaluated. In Fig. 7, the 41-s-average horizontal wind speeds obtained from the ultrasonic anemometer are plotted in black and the 10-min-average horizontal speeds obtained from the WindScanner system are plotted in red. The gray area is based on the maximum and minimum of the WindScanner-measured 12-s-average horizontal speeds in each grid cell obtained during the 10-min-long measurement period. The agreement between the two instruments is good, and the slightly higher speeds measured by the ultrasonic anemometer close to the helicopter center could be attributed to the sonic anemometer alignment, causing part of the large vertical wind component present close to the helicopter to show up as a horizontal wind component.

\section{Discussion and conclusions}

The complex and turbulent downwash flow field below a SAR helicopter in this study has been characterized with high spatial resolution remotely by two short-range WindScanners. The results suggest that the continuous-wave Doppler-lidar-based WindScanner technology can assess downwash and potentially support rotorcraft optimization regarding, for example, minimizing the risk to aircraft and personnel when operating in a search and rescue role, and can contribute to the understanding about brownout and similar phenomena.
The current implementation of the technology can measure the line-of-sight velocity in the range from -21 up to $18 \mathrm{~m} \mathrm{~s}^{-1}$. However, this velocity range is not a fundamental limit, since it can be extended by, for example, a higher center frequency shift combined with a higher sampling rate or by introducing a quadrature detection scheme (Hogenboom and DiMarzio 1998), which can provide the sign of the line-of-sight velocity without the use of an acousto-optic modulator.

Combining the flow field measurement capabilities of the WindScanner technology with the concentration mapping capabilities provided by scanning aerosol and differential absorption lidars could constitute a fruitful combination for detailed studies of rotorcraft-downwashgenerated brownout as well as remote flux estimation of environmental pollutants (Sjöholm et al. 2004).

These first mean two-dimensional complex turbulent wind field measurements by the WindScanner technology are not only of interest to the aviation community. In fact, the technology also has applications within the wind energy and atmospheric boundary layer communities, since the spatial and temporal resolution provided by the WindScanners is suitable for studying

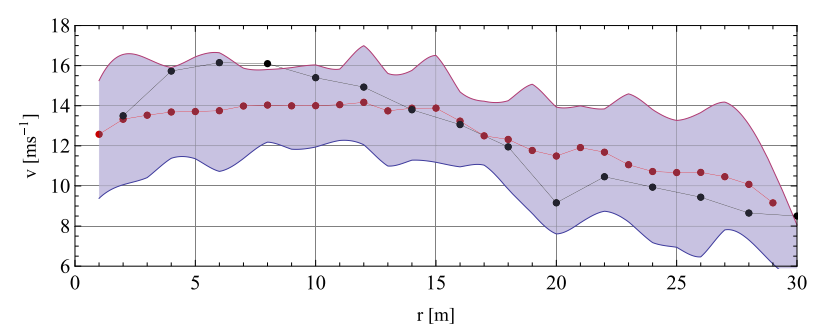

FIG. 7. The 41-s-average horizontal wind speeds obtained from the ultrasonic anemometer (black) and the 10-min-average ones from the WindScanner system (red). Also plotted is the maximum and minimum of the WindScanner-measured 12-s-average horizontal speeds in each grid cell obtained during the 10-min-long measurement period (gray area). 
detailed flow fields, mean and turbulent, on scales typically present around wind turbines, buildings, and small natural structures.

Acknowledgments. The authors gratefully acknowledge the financial support from the Danish Agency for Science, Technology and Innovation through Grant 2136-08-0022 for the Danish research infrastructure facility WindScanner.dk (http://www.windscanner.dk).

\section{REFERENCES}

Angelou, N., F. Foroughi Abari, J. Mann, T. Mikkelsen, and M. Sjöholm, 2012a: Challenges in noise removal from Doppler spectra acquired by a continuous-wave lidar. Proc. 26th Int. Laser Radar Conf., Porto Heli, Peloponnesus, Greece, ILRC, S5P-01. [Available online at http://orbit.dtu.dk/fedora/objects/ orbit:113163/datastreams/file_9824562/content.]

— J. Mann, M. Sjöholm, and M. Courtney, 2012b: Direct measurement of the spectral transfer function of a laser based anemometer. Rev. Sci. Instrum., 83, 033111, doi:10.1063/ 1.3697728.

Fiorino, S. T., J. A. Deibel, P. M. Grice, M. H. Novak, J. Spinoza, L. Owens, and S. Ganti, 2012: A technique to measure optical properties of brownout clouds for modeling terahertz propagation. Appl. Opt., 51, 3605-3613.

Gottschall, J., M. S. Courtney, R. Wagner, H. E. Jorgensen, and I. Antoniou, 2012: Lidar profilers in the context of wind energy-A verification procedure for traceable measurements. Wind Energy, 15, 147-159, doi:10.1002/we.518.

Hogenboom, D., and C. DiMarzio, 1998: Quadrature detection of a Doppler signal. Appl. Opt., 37, 2569-2572.
Jansen, C., A. Wennemers, W. Vos, and E. Groen, 2008: Flytact: A tactile display improves a helicopter pilot's landing performance in degraded visual environments. Haptics: Perception, Devices and Scenarios, M. Ferre, Ed., Lecture Notes in Computer Science, Vol. 5024, Springer, 867-875.

Karlsson, C. J., F. A. Olsson, D. Letalick, and M. Harris, 2000: Allfiber multifunction continuous-wave coherent laser radar at $1.55 \mu \mathrm{m}$ for range, speed, vibration, and wind measurements. Appl. Opt., 39, 3716-3726, doi:10.1364/AO.39.003716.

Mikkelsen, T., J. Mann, M. Courtney, and M. Sjöholm, 2008: Windscanner: 3-D wind and turbulence measurements from three steerable Doppler lidars. IOP Conf. Ser.: Earth Environ. Sci., 1, 012018, doi:10.1088/1755-1315/1/1/012018.

Milluzzo, J., and J. G. Leishman, 2010: Assessment of rotorcraft brownout severity in terms of rotor design parameters. J. Amer. Helicopter Soc., 55, 032009, doi:10.4050/JAHS.55.032009.

Newsom, R., R. Calhoun, D. Ligon, and J. Allwine, 2008: Linearly organized turbulence structures observed over a suburban area by dual-Doppler lidar. Bound.-Layer Meteor., 127, 111130, doi:10.1007/s10546-007-9243-0.

Roy, G., X. Cao, S. Roy, and E. Trickey, 2012: Assessment of helicopter brownout with a scanning lidar. Conf. on Lasers and Electro-Optics (CLEO), San Jose, CA, IEEE, ATu2G.5, doi:10.1364/CLEO_AT.2012.ATu2G.5.

Sjöholm, M., P. Weibring, H. Edner, and S. Svanberg, 2004: Atomic mercury flux monitoring using an optical parametric oscillator based lidar system. Opt. Express, 12, 551-556.

— - T. Mikkelsen, J. Mann, K. Enevoldsen, and M. Courtney, 2009: Spatial averaging-effects on turbulence measured by a continuous-wave coherent lidar. Meteor. Z., 18, 281-287.

Wadcock, A., L. Ewing, E. Solis, M. Potsda, and G. Rajagopalan, 2008: Rotorcraft downwash flow field study to understand the aerodynamics of helicopter brownout. American Helicopter Society Southwest Region Specialists Meeting on Next Generation Vertical Lift Technologies, AHSI, 528-554. 